\title{
Micronized and Heat-Treated Lactobacillus plantarum LM1004 Stimulates Host Immune Responses Via the TLR-2/MAPK/NF- $\kappa B$ Signalling Pathway In Vitro and In Vivo
}

\author{
Jisun Lee ${ }^{1}$, Ilseon Jung ${ }^{2}$, Ji Won Choi ${ }^{1}$, Chang Won Lee ${ }^{1}$, Sarang Cho ${ }^{1}$, Tae Gyu Choi ${ }^{3}$, Minn Sohn ${ }^{2}$, and \\ Yong Il Park ${ }^{1 *}$ \\ ${ }^{1}$ Department of Biotechnology, The Catholic University of Korea, Bucheon 14662, Republic of Korea \\ ${ }^{2}$ LACTOMASON, Jinju 52840, Republic of Korea \\ ${ }^{3}$ Department of Biochemistry and Molecular Biology, School of Medicine, Kyung Hee University, Seoul 02447, Republic of Korea
}

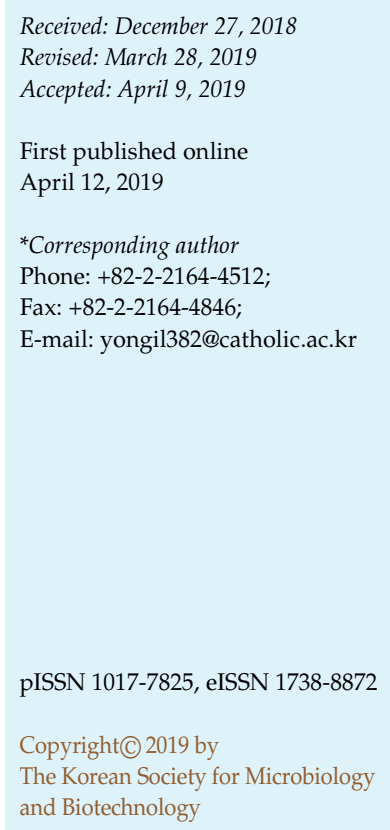

\begin{abstract}
Although nanometric dead Lactobacillus plantarum has emerged as a potentially important modulator of immune responses, its underlying mechanism of action has not been fully understood. This study aimed to identify the detailed biochemical mechanism of immune modulation by micronized and heat-treated L. plantarum LM1004 (MHT-LM1004, <1 $\mu \mathrm{m}$ in size). MHT-LM1004 was prepared from L. plantarum LM1004 via culture in a specifically designed membrane bioreactor and heat treatment. MHT-LM1004 was shown to effectively induce the secretion of TNF- $\alpha$ and IL- 6 and the mRNA expression of inducible nitric oxide synthase (iNOS). MHT-LM1004 enhanced the expression of TLR-2, phosphorylation of MAPKs (ERK), and nuclear translocation of NF- $\mathrm{B}$ in a dose-dependent manner. Oral administration of MHT-LM1004 $\left(4 \times 10^{9}\right.$ or $4 \times 10^{11}$ cells $/ \mathrm{kg}$ mouse body weight $)$ increased the splenocyte proliferation and serum cytokine levels. These results suggested that MHT-LM1004 effectively enhances early innate immunity by activating macrophages via the TLR-2/MAPK/NF- $B$ signalling pathway and that this pathway is one of the major routes in immune modulation by the Lactobacillus species.
\end{abstract}

Keywords: Nanometric heat-killed Lactobacillus plantarum, immunostimulating activity, action mechanism, TLR-2, MAPK-NF-kB signalling pathway

\section{Introduction}

Probiotics are generally defined as live microorganisms, such as Lactobacillus and Bifidobacteria, which confer a beneficial health effect to the host when administered in adequate amounts [1]. It has been reported that probiotics have a wide range of biological activities including immune regulation, improvement in the intestinal environment, and cancer prevention [2,3]. Most studies on the beneficial effects of probiotics have focused on viable microorganisms. However, it has been reported that there are several problems in the practical applications such as heterogeneity of live probiotics and low survival rate in the gastrointestinal tract due to low $\mathrm{pH}$ conditions and rich proteolytic enzymes [2]. Interestingly, emerging evidence from various studies has revealed that even heat-treated probiotics might confer apparent health benefits to the host even though the FAO/ WHO still emphasize a limited use of live microorganisms for probiotics [4, 5]. It was reported that the thermal treatments increase the cell coarseness and roughness, which influence their immune-modulating properties [6, 7]. In addition, heat-treated probiotics not only possess biological activities in communication with the host but also provide advantages for industrial production and consumer usage, including a longer product shelf life, easier storage, and more convenient transportation $[4,6,8]$. 
On the other hand, it was reported that nanometric Lactobacillus plantarum (nLp) showed an improved ability to induce interleukin-12 (IL-12), suggesting that micronized L. plantarum more effectively modulates the host immune system than intact L. plantarum $[9,10]$. nLp was manufactured by incubating live L. plantarum under specified stress conditions, such as incubation at a high temperature, starvation, high salinity, or low $\mathrm{pH}$, which cause cells to become rounder and smaller $(<1 \mu \mathrm{m})$ than those of rod-like, live L. plantarum cells [10]. It was also reported that smaller antigens are more rapidly taken-up by $\mathrm{M}$ cells in the Peyer's patches and induce greater IgA secretion [11]. Furthermore, it was reported that micronized L. plantarum showed stronger anti-colitis and anti-colorectal cancer effects than intact L. plantarum in a mouse model $[9,12]$. Thus, industrial production of heattreated and/or micronized (smaller-sized) probiotics is attracting more attention for manufacturing better probiotics products with enhanced health benefits including immunomodulatory functions. However, although these heat-killed and micronized microorganisms possess enhanced immunomodulatory activities, their detailed action mechanisms in immune modulation have not been extensively explored.

Previously, a strain of L. plantarum from Korean kimchi (fermented napa cabbage) was isolated and named L. plantarum LM1004 (KCCM 4246). Subsequently, we manufactured its micronized and heat-treated form (named as MHT-LM1004) by incubating live L. plantarum LM1004 under physically harsh conditions in a specifically designed membrane bioreactor (MBR) (Korea Patent 10-1245208). The MHT-LM1004 is a dead, shrunken, granular and smaller (less than $0.5-1.0 \mu \mathrm{m}$ in size) form of rod-like, live L. plantarum LM1004. In this study, whether the MHTLM1004 induces the immune response, both in vitro, and in vivo and its detailed biochemical mechanism of immunostimulating activity were investigated.

\section{Materials and Methods}

\section{Materials}

Fetal bovine serum (FBS), penicillin-streptomycin, and trypsinEDTA were purchased from Lonza (USA). 3-(4,5-dimethylthiazolyl)-diphenyl tetrazolium bromide (MTT) was provided by Duchefa Biochemie (Netherlands). Dulbecco's Modified Eagle's Medium (DMEM) and RPMI-1640 medium (RPMI) were purchased from WelGENE (Korea). The antibodies against total ERK ( $\mathrm{t}$-ERK), phospho-ERK (p-ERK), NF-кB p65, $\beta$-actin, goat anti-mouse IgGHRP and goat anti-rabbit IgG-HRP were obtained from Cell Signaling Technology (USA).

\section{Preparation of MHT-LM1004}

Micronized and heat-treated L. plantarum LM1004 (MHTLM1004) was prepared from L. plantarum LM1004 (KCCM 4246), which was isolated from Korean kimchi and filed by LactoMason (Korea). Briefly, the live, rod-like L. plantarum LM1004 was cultivated at $32^{\circ} \mathrm{C}$ and $\mathrm{pH} 6.0$ in a specifically designed membrane bioreactor (MBR) in our laboratory (KR Patent 10-1245208). High cell-density cultures and the cell size control for manufacturing of MHT-LM1004 were conducted under shear stress conditions, controlling the pump speed and the flow rate of the cell culture fluids in the MBR (15-L pilot scale, anaerobic conditions with $\mathrm{N}_{2}$ gas), which was externally fitted with the ceramic membrane (5.7- $\mathrm{m}^{2}$ effective area, $0.2-\mu \mathrm{m}$ pore size, Pall Corporation, USA) and the centrifugal pump (Grundfos, Denmark). The wall shear stress, which is one of the crucial factors for altering the morphology of L. plantarum LM1004, was generated while the cells were passing through the hydraulic channels of the ceramic membrane and the centrifugal pump [13, 14]. After intensive propagation in the MBR, the cells were killed at $80^{\circ} \mathrm{C}$ for $20 \mathrm{~min}$ (Ultra High Temperature, Sam-Ryung Sterilization Co, Korea), washed through the ultrafiltration membrane (effective area of $1.2 \mathrm{~m}^{2}, 0.1-\mu \mathrm{m}$ pore size, Pall Corporation) three times with reverse osmosis (RO) water, and freeze dried. The morphologies and particle distribution $(<1 \mu \mathrm{m}$ in size) of MHT-LM1004 cells were determined using field-emission scanning electron microscopy (JSM-7610F, JEOL, Japan) and particle size analyzer (1090LD, CILAS, France), respectively.

\section{Cell Culture}

RAW 264.7 macrophage cells were purchased from the Korea Cell Line Bank (Korea) and grown in DMEM supplemented with $10 \%(\mathrm{v} / \mathrm{v})$ FBS, $2 \mathrm{mM}$ L-glutamine and $100 \mathrm{U} / \mathrm{ml}$ penicillinstreptomycin. The cytotoxicity of MHT-LM1004 was assessed by measuring cell viability using the MTT assay [15]. To examine the role of TLR-2 in cytokine production, RAW 264.7 cells were preincubated with medium containing the TLR-2-specific antibody $(10 \mu \mathrm{g} / \mathrm{ml})$ or IgG1 antibody (isotype control antibody, $10 \mu \mathrm{g} / \mathrm{ml}$ ) (Invivogen, USA) for $1 \mathrm{~h}$. Cells were then treated with MHTLM1004 $\left(10^{7}\right.$ cells $\left./ \mathrm{ml}\right)$ for $4 \mathrm{~h}$.

\section{Assay for TNF- $\alpha$ and IL-6 Secretion}

The cells were cultured in DMEM for $24 \mathrm{~h}$ with various concentrations of MHT-LM1004 $\left(10^{2} \sim 10^{7}\right.$ cells $\left./ \mathrm{ml}\right)$ and the supernatants were used for the quantification of TNF- $\alpha$ and IL-6 secretion using an ELISA kit (R\&D Systems, United Kingdom) according to the manufacturer's protocol.

\section{Measurement of NO Production}

The RAW 264.7 cells were cultured for $24 \mathrm{~h}$ in DMEM supplemented with $10 \%$ FBS and 1\% PEST. The cells were then treated with different concentrations of MHT-LM1004 $\left(10^{3} \sim 10^{7}\right.$ cells $/ \mathrm{ml}$ ) for $24 \mathrm{~h}$. NO levels in the culture supernatants were determined using Griess reagent (Sigma, USA). Briefly, samples of 
the culture supernatants were mixed with an equal volume of Griess reagent $[0.1 \%$ naphthyl ethylenediamine $(\mathrm{w} / \mathrm{v})$ and $1 \%$ sulfanilamide $(\mathrm{w} / \mathrm{v})$ in $5 \%$ phosphoric acid $(\mathrm{v} / \mathrm{v})]$, and the absorbance at $540 \mathrm{~nm}$ was measured. Sodium nitrite $\left(\mathrm{NaNO}_{2}\right.$, Sigma) was used as a reference.

\section{Reverse Transcriptase-Polymerase Chain Reaction (RT-PCR) and Western Blot Analysis}

To evaluate the mRNA expression levels of iNOS, TLR-2 and TLR-4, the total RNA from MHT-LM1004-treated RAW 264.7 cells was prepared using a Total RNA Extraction Kit (Intron Biotechnology, Korea). RT-PCR was performed using the OneStep RT-PCR PreMix Kit (Intron Biotechnology) with appropriate sense and antisense. The products were separated on a $1.5 \%$ agarose gel, stained with ethidium bromide and then viewed under UV transillumination. Western blot was performed as described previously [16]. The mRNA and protein were quantified using ImageJ software from the NIH (USA).

\section{Immunocytochemistry}

Cells were grown on an 8-chamber slide, fixed with $4 \%$ formaldehyde in PBS, and then permeabilized and blocked for $1 \mathrm{~h}$ with blocking buffer ( $5 \%$ normal goat serum and $0.3 \%$ Triton $\mathrm{X}$ 100 in PBS). For NF-кB visualizations, cells were incubated overnight with primary antibody and diluted 1:100 in antibody dilution buffer (1\% BSA and 0.3\% Triton X-100 in PBS). Cells were then incubated for $2 \mathrm{~h}$ with fluorochrome-conjugated secondary antibody, diluted 1:1,000, and then washed three times with PBS. The cover glass was applied to the slide with Prolong Gold Antifade Reagent with DAPI (all from Cell Signaling Technology). The stained cells were monitored using a Zeiss LSM800 confocal laser scanning microscope (Carl Zeiss, Germany, X630).

\section{Animals}

The animal protocol (Approval number: 2016-021) was approved by the Institutional Animal Care and Use Committee of The Catholic University of Korea. The 6-week-old female BALB/c mice were purchased from Orientbio (Korea) and randomly divided into three equal groups ( $n=6$ in each group): control group (Con) not receiving the MHT-LM1004 solution, and two experimental groups who were orally administered the MHTLM1004 solution at the concentrations of $4 \times 10^{9}$ or $4 \times 10^{11}$ cells $/ \mathrm{ml}$. After 28 days of administration of MHT-LM1004, the mice were sacrificed, and their blood ( $n=5$ in each group) and spleens ( $n=6$ in each group) were individually taken for further analyses.

\section{Isolation of Splenocytes}

To isolate splenocytes, the tissues were disaggregated via passage through a 70- $\mu$ m nylon mesh (Becton-Dickinson, USA) in RPMI. The cells were isolated via centrifugation at $470 \mathrm{~g}$ for $10 \mathrm{~min}$, and red blood cells (RBC) were removed with a lysis buffer (Sigma-Aldrich). Splenocytes were then washed with PBS and centrifuged at $470 \mathrm{~g}$ for $5 \mathrm{~min}$ and finally suspended in RPMI supplemented with 10\% (v/v) FBS, 2 mM L-glutamine and 100 U/ml penicillin-streptomycin.

\section{Measurement of Splenocyte Proliferation Rate and Serum Cytokine}

The splenocytes were suspended in RPMI supplemented with $10 \%(\mathrm{v} / \mathrm{v}) \mathrm{FBS}$ and dispensed into 12 -well plates $\left(5 \times 10^{6}\right.$ cells $\left./ \mathrm{ml}\right)$. Cells were then incubated in RPMI containing mitogens concanavalin A (ConA, $5 \mu \mathrm{g} / \mathrm{ml}$, Sigma-Aldrich) as a T-cell mitogen or E. coli lipopolysaccharide (LPS, $15 \mu \mathrm{g} / \mathrm{ml}$, SigmaAldrich) as a B-cell mitogen for $48 \mathrm{~h}$. After incubation, the proliferation rate of splenocytes from the mice administered MHT-LM1004 was assessed by measuring cell viability using the MTT assay and expressed as the percentage normalized to the untreated control cells (Con). Splenocytes isolated from six individuals of each group were used for the MTT assay. The serum cytokine levels (TNF- $\alpha$ and IL-6) were determined using ELISA kits (R\&D Systems, United Kingdom).

\section{Statistical Analysis}

Measurements were performed in triplicate, and data are expressed as the means $\pm \mathrm{SD}$. The statistical analyses were performed using the unpaired Student's $t$-test (in vitro tests) and Statistical Analysis Systems software package (in vivo tests) (SAS Institute, USA). A $p$-value less than 0.05 was considered statistically significant.

\section{Results}

\section{Preparation of MHT-LM1004}

Micronized and heat-treated L. plantarum LM1004 (MHTLM1004) was prepared by culturing rod-like, live L. plantarum LM1004 (KCCM 4246) in a specifically designed membrane bioreactor (MBR) under high shear stress conditions and then heat treatment at $80^{\circ} \mathrm{C}$ for $20 \mathrm{~min}$. As shown in Fig. 1A, the morphology of the resulting cells was shown to be shrunken and granular compared to that of the rod-like L. plantarum LM1004. The particle size (in diameter) distribution analysis also showed that the cell size was reduced to less than $1.0 \mu \mathrm{m}$ in response to the wall shear stress given to the cells while growing them in the MBR [17].

\section{Effect of MHT-LM1004 on the Viability of RAW 264.7 Cells}

To determine whether MHT-LM1004 is detrimental to RAW 264.7 cells, the effects of MHT-LM1004 on the viability were tested using the MTT assay after treatment with increasing concentrations of MHT-LM1004 for $24 \mathrm{~h}$. MHT-LM1004 did not affect the viability of RAW 264.7 cells up to the concentrations of $10^{8}$ cells $/ \mathrm{ml}$ (Fig. 1B), showing that MHT-LM1004 has no cytotoxicity on RAW 264.7 cells. 
A
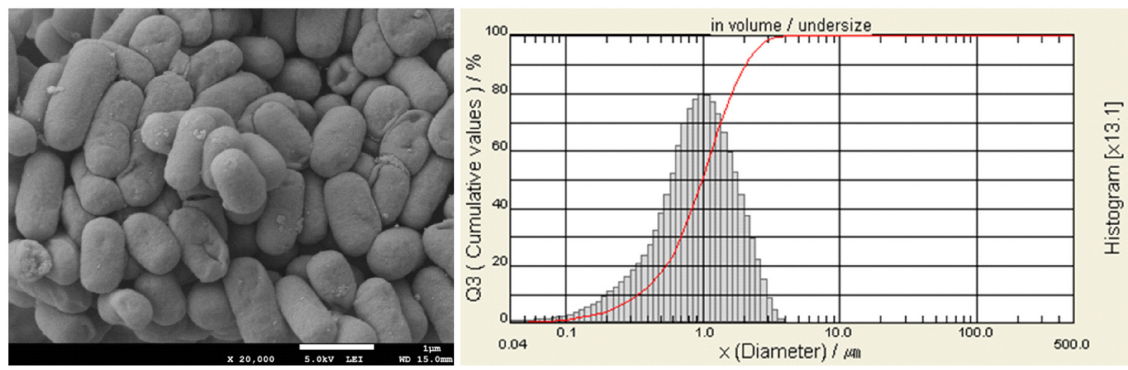

B

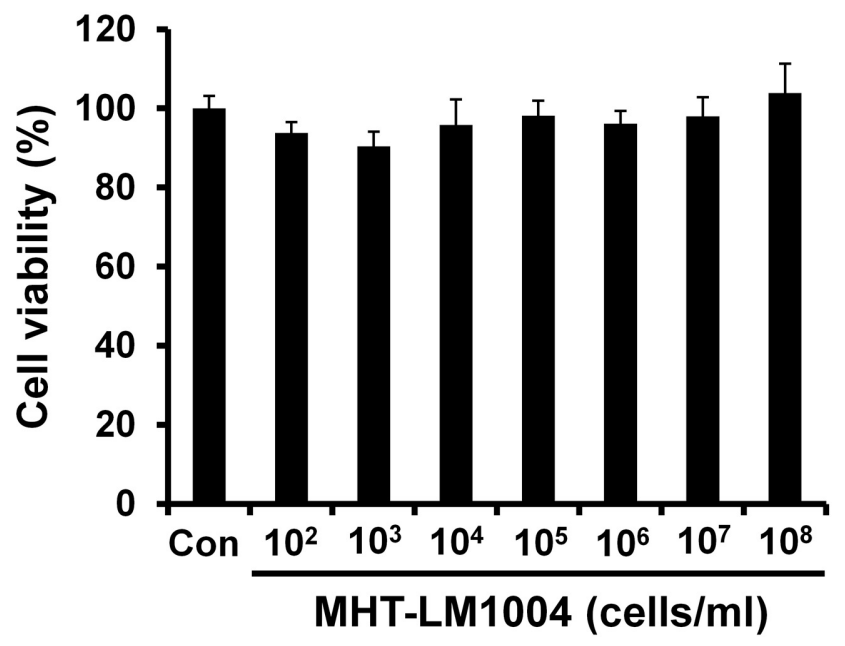

Fig. 1. Effects of MHT-LM1004 on the viability of RAW 264.7 cells.

(A) Scanning electron micrograph (left) and particle size distribution (right) of MHT-LM1004. (B) Cell viability was assessed using the MTT assay after treatment with LM1004 for $24 \mathrm{~h}$. The data are expressed as the percentage normalized to the untreated control (Con); Data $=$ the means \pm SD, $n=3$.

Thus, concentrations of less than $10^{8}$ cells/ml of the MHTLM1004 were used for subsequent experiments to evaluate its immunostimulatory activities.

\section{Effects of MHT-LM1004 on Cytokine Production}

Activated macrophages release pro-inflammatory cytokines such as TNF- $\alpha$ and IL-6, which are potent immunomodulators [16, 18]. As shown in Fig. 2, MHT-LM1004 significantly induced TNF- $\alpha$ (Fig. 2A) and IL-6 (Fig. 2B) secretion in a dose-dependent manner. MHT-LM1004 treatment resulted in approximately 24.9-fold and 19.3-fold higher levels of TNF- $\alpha$ and IL-6, respectively, at $10^{7}$ cells $/ \mathrm{ml}$ compared to the untreated control (Con), suggesting that MHT-LM1004 is a strong stimulator of TNF- $\alpha$ and IL-6 secretion.

\section{Effects of MHT-LM1004 on iNOS Expression and NO Production}

NO production plays a crucial role in the immune response [19]. When the RAW 264.7 cells were incubated with MHT-LM1004 for $24 \mathrm{~h}$, the iNOS expression was significantly enhanced (Fig. 3A), with approximately 17.7fold higher expression at $10^{7}$ cells $/ \mathrm{ml}$ MHT-LM1004, compared to the untreated cells (Con) (Fig. 3B). This result was further confirmed by measuring the level of $\mathrm{NO}$ production. When the cells were treated with varying concentrations of MHT-LM1004 $\left(10^{3} \sim 10^{7}\right.$ cells $\left./ \mathrm{ml}\right)$ for $24 \mathrm{~h}$, the levels of NO were significantly enhanced in a dose-dependent manner (Fig. 3C). These results suggested that NO is a mediator of MHT-LM1004-stimulated immune signalling pathways.

\section{MHT-LM1004 Induces Cytokine Production Dependent on TLR-2 Gene Expression}

Toll-like receptors (TLRs) are pattern recognition receptors that have important functions in the initiation of mammalian immune responses [20]. Thus, to understand how the MHT-LM1004 affects the innate immune response, responsible receptors on the surfaces of macrophages were 

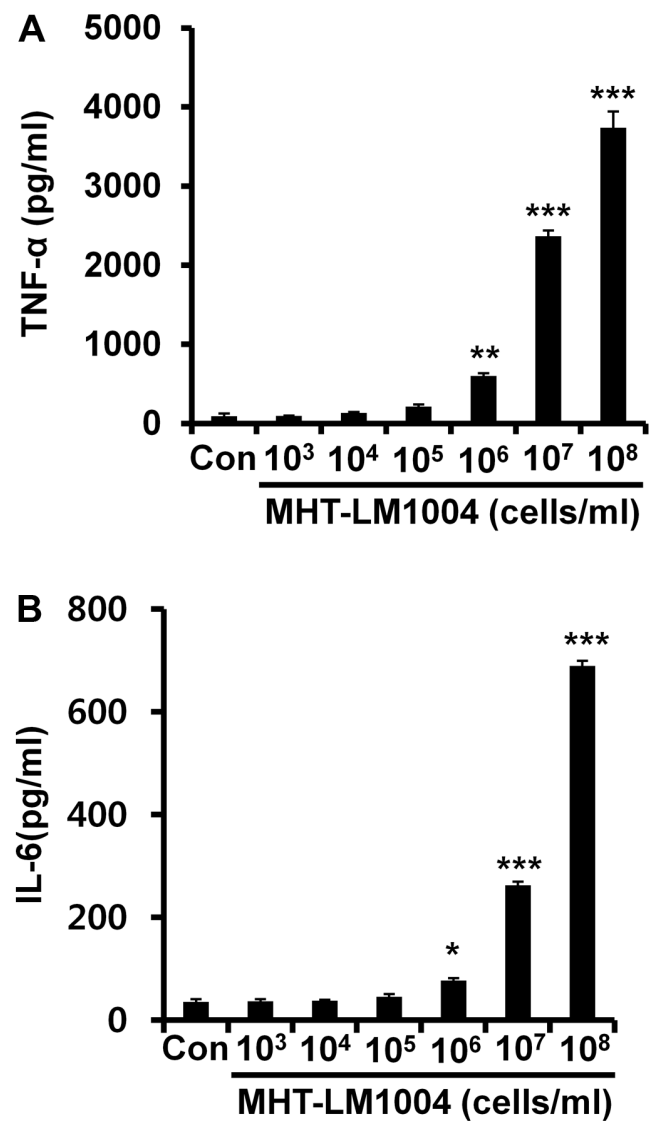

Fig. 2. Effects of MHT-LM1004 on TNF- $\alpha$ and IL-6 secretion. Cells were incubated with the indicated doses of MHT-LM1004 for $24 \mathrm{~h}$. The levels of (A) TNF- $\alpha$ and (B) IL- 6 in the culture supernatants were determined using ELISA. Data $=$ mean $\pm \mathrm{SD}, n=3 ;{ }^{*}, p<0.05$; **, $p<0.01 ; * * *, p<0.001$, Student's $t$-test compared to the untreated control (Con)

monitored. The RAW 264.7 cells were treated with MHTLM1004 for $24 \mathrm{~h}$, and then the mRNA expression levels of the candidate membrane receptors including TLR-2 and TLR-4 were determined using RT-PCR. As shown in Fig. 4A, MHT-LM1004 treatment resulted in a remarkable increase of TLR-2 mRNA expression levels, whereas TLR-4 expression levels did not change. This result was further confirmed using anti-TLR-2 antibody. The treatment of macrophages with the TLR-2 antibody significantly reduced MHT-LM1004-induced up-regulation of TNF- $\alpha$ secretion (Fig. 4B), clearly demonstrating that the target receptor of MHT-LM1004 was TLR-2 on the RAW 264.7 cells.

MHT-LM1004 Stimulates the Immune System Via the MAPKs/NF- $\kappa$ B Pathway

Interestingly, the TLR-2 activation recruits the myeloid differentiation primary response gene 88 (MyD88) adapter
A

MHT-LM1004 (cells/ml)
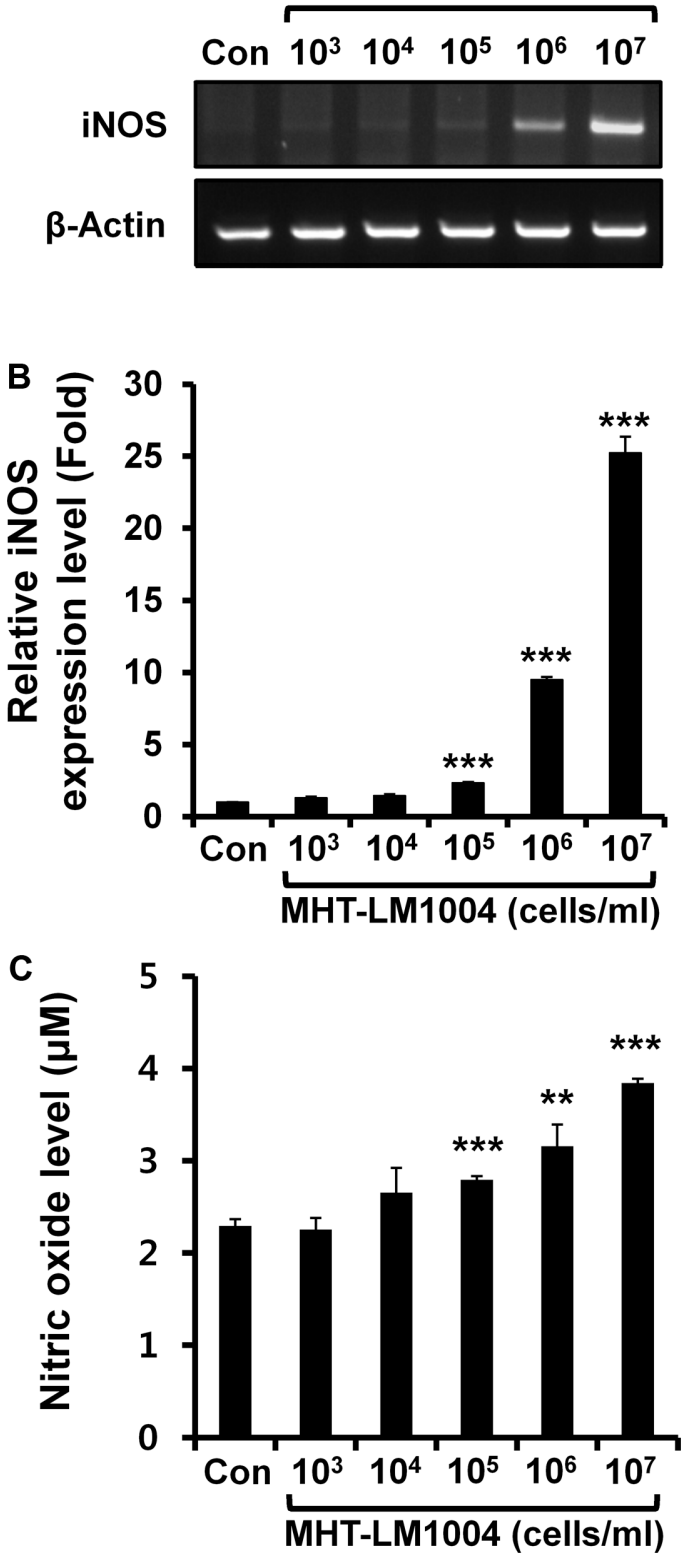

Fig. 3. Effects of MHT-LM1004 on iNOS mRNA expression. (A) The mRNA level of iNOS was determined using RT-PCR. (B) The amount of iNOS mRNA is expressed as the ratio of the densitometric measurement of mRNA in MHT-LM1004-treated cells to the corresponding internal standard ( $\beta$-Actin). (C) Nitric oxide levels in the culture media of MHT-LM1004-stimulated cells were measured using the Griess reagent. Data $=$ mean $\pm \mathrm{SD}, n=3 ; * *, p<0.01$; ***, $p<0.001$, Student's $t$-test compared to the untreated control (Con).

protein and activates MAPK signalling pathways including extracellular-signal-regulated kinase (ERK) for regulating the immune response in macrophages [16, 20, 21]. The western blot analysis showed that while the total protein 
A

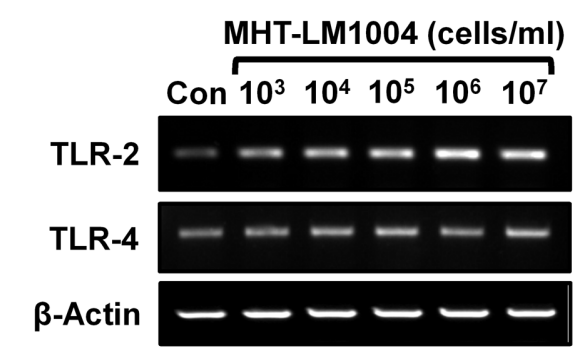

B

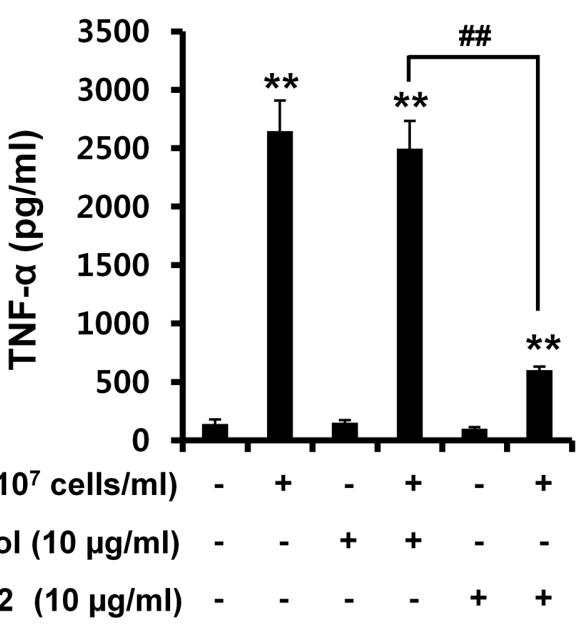

Fig. 4. MHT-LM1004 induces TNF- $\alpha$ production through TLR-2 gene expression in RAW 264.7 cells.

(A) RAW 264.7 cells were incubated with the indicated doses of MHT-LM1004 for $24 \mathrm{~h}$. The mRNA expression levels of TLR-2 and TLR-4 were measured using RT-PCR. (B) Cells were pre-treated with isotype antibody $(10 \mu \mathrm{g} / \mathrm{ml})$ or anti-TLR-2 antibody $(10 \mu \mathrm{g} / \mathrm{ml})$ for 1 $\mathrm{h}$ and then stimulated with MHT-LM1004 ( $10^{7}$ cells $\left./ \mathrm{ml}\right)$ for $4 \mathrm{~h}$. TNF$\alpha$ in the culture supernatants was determined using ELISA. Data = mean $\pm \mathrm{SD}, n=3 ;{ }^{* *}, p<0.01$, Student's $t$-test compared to the control without MHT-LM1004 or antibody-only treated cells; \#\#, $p<0.01$, Student's $t$-test compared to the MHT-LM1004 plus isotype antibody treated cells.

levels of ERK (t-ERK) remained unchanged, the treatment of cells with MHT-LM1004 for $24 \mathrm{~h}$ significantly increased the phosphorylation levels of ERK (Fig. 5A), suggesting that MHT-LM1004 activates the TLR-2/MAPKs (ERK) signalling pathway to enhance the immune response in RAW 264.7 cells. On the other hand, NF-KB is known as a key transcription factor, which regulates immune response genes. The expression of iNOS is directly coupled to the up-regulation of NF-кB [22]. The immunocytochemistry result showed that MHT-LM1004 treatment increased the translocation of NF- $\mathrm{KB}$ from the cytosol to the nucleus compared to the untreated control (Con) (Fig. 5B). Taken collectively, these data clearly demonstrated that MHT-
A

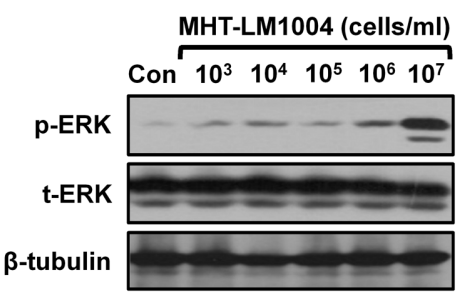

B

DAPI p65
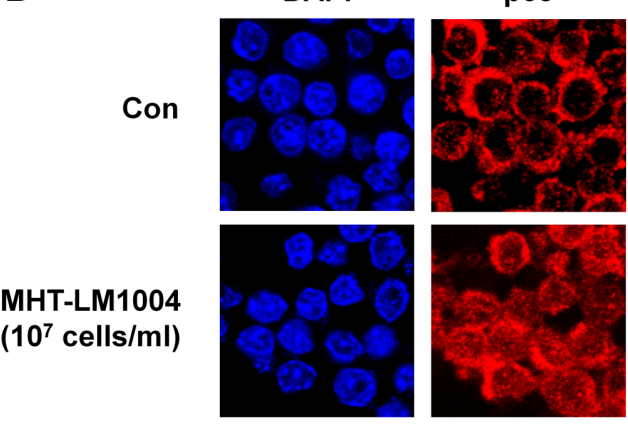

Merge
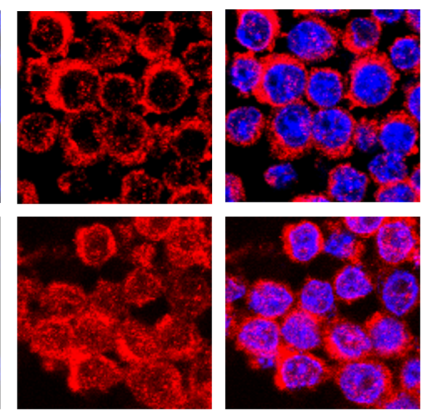

Fig. 5. MHT-LM1004 activates the MAPKs/NF-kB pathway in RAW 264.7 cells.

(A) Whole cell lysates were collected after treatment with MHTLM1004 for $24 \mathrm{~h}$. t-ERK was used as a loading control for the western blot analysis of $\mathrm{p}$-ERK. (B) The cells were exposed to MHT-LM1004 $\left(10^{7}\right.$ cells $\left./ \mathrm{ml}\right)$ for $24 \mathrm{~h}$, stained with DAPI solution and anti-NF- $\mathrm{kB}$ p65 (Rhodamine) antibody, and then visualized under a Zeiss LSM800 confocal laser scanning microscope (Carl Zeiss, Germany, X630).

LM1004 activates the TLR-2/MAPK/NF-kB pathway in RAW 264.7 cells for immune modulation.

Effects of MHT-LM1004 on the Splenocyte Proliferation Rate and Cytokine Production In Vivo

To prove if MHT-LM1004 is equally effective in vivo, the mice were fed with MHT-LM1004 $\left(4 \times 10^{9}\right.$ or $4 \times 10^{11}$ cells $\left./ \mathrm{ml}\right)$ for 28 days, and the splenocytes were isolated from the spleens of the mice and then treated with mitogens (ConA or LPS). As shown in Figs. 6A and 6B, ConA and LPS treatment increased the proliferation of splenocytes isolated from the mice treated with $4 \times 10^{11}$ cells $/ \mathrm{ml}$ MHTLM1004 up to 2.3-fold and 1.6-fold, respectively, $(p<0.05)$ compared to that of the control group stimulated with mitogen alone (ConA and LPS), showing that MHTLM1004 enhances the proliferation of splenocytes in vivo. In addition, the serum levels of TNF- $\alpha$ and IL- 6 cytokines were increased in MHT-LM1004-treated groups compared to that of the control group (Con) (Figs. 6C and 6D). These results suggested that MHT-LM1004 positively stimulates the immune system both in vitro and in vivo. 

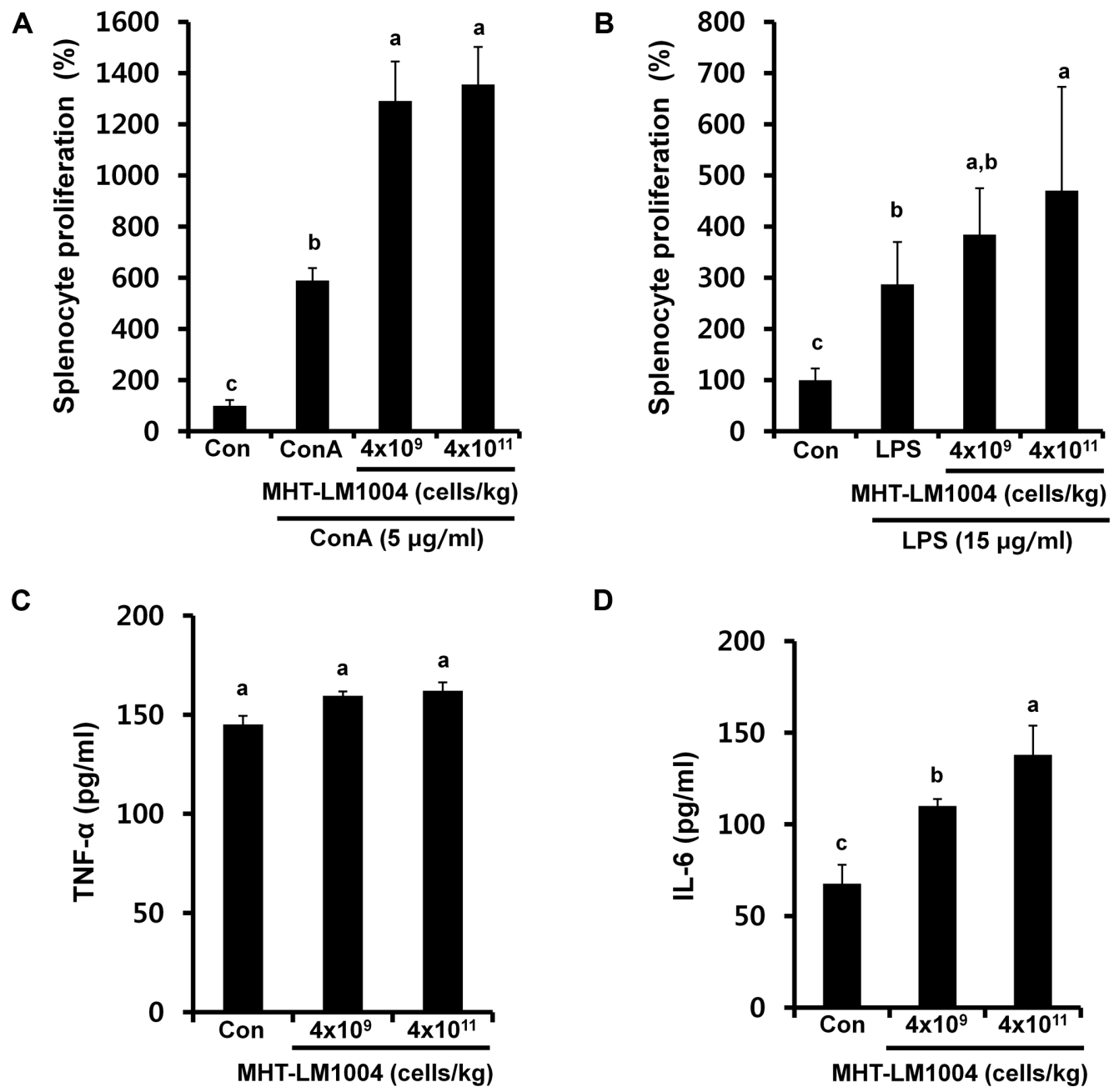

Fig. 6. Effects of MHT-LM1004 on the splenocyte proliferation rate and serum cytokine production in vivo.

(A and B) Splenocytes $\left(5 \times 10^{6}\right.$ cells per well) were individually isolated from the BALB/c mice orally administered with MHT-LM1004 for 28 days and cultured with mitogens (ConA or LPS) for $48 \mathrm{~h}(n=6$ in each group). The proliferation of splenocytes was determined using the MTT assay. The data are expressed as the percentage normalized to the untreated control cells (Con). (C and D) Blood was withdrawn via cardiac puncture from the mice orally administered MHT-LM1004 for 28 days, and serum TNF- $\alpha$ and IL- 6 was measured using the ELISA kit ( $n=5$ in each group). All values are expressed as the mean \pm SE. Values with different superscripts are significantly different among the groups according to ANOVA Duncan's multiple range test at $p<0.05$.

\section{Discussion}

Generally, it is well known that heat-killed probiotics, such as Enterococcus faecalis or Bifidobacteria, and fractionated cell components, such as cell wall preparations from E. faecalis or Lactobacillus spp. or lipoteichoic acids from L. casei or L. fermentum, augment host resistance and stimulate the innate immune response $[2,12]$. However, the underlying action mechanism of their immune modulating activities is still unclear. Previous studies suggested a possible mechanism whereby probiotics are taken up by $\mathrm{M}$ cells and directly affect Peyer's patch immunocompetent cells to stimulate intestinal immune responses [9, 23]. Despite the number of extensive studies performed focusing on live probiotics, there has not been enough evidence for their exact bioavailability.

Here, we focused on the advantages of heat-treated probiotics, such as a longer shelf-life and enhanced immunomodulatory activities. When the live L. plantarum LM1004 was cultured under high shear stress conditions in the membrane bioreactor (MBR) and then heat-treated, the cells became shrunken, granular and smaller (less than 
$1.0 \mu \mathrm{m})$ than the rod-like L. plantarum LM1004. The resulting micronized and heat-treated L. plantarum LM1004 (MHT-LM1004) was shown to enhance the splenocyte proliferation rate and production of proinflammatory cytokines, which indicates that MHT-LM1004 effectively stimulates immune responses in vivo. In accordance with our results, it was demonstrated that the micronized L. plantarum stimulated splenocyte proliferation more than intact L. plantarum in vitro [12]. Furthermore, the micronized L. plantarum could inhibit colon carcinogenesis through stronger intestinal immune responses than the intact L. plantarum; it was suggested that these activities of the micronized L. plantarum were associated with its easier uptake by $\mathrm{M}$ cells than the intact $L$. plantarum because of its smaller particle size (less than $1 \mu \mathrm{m}$ ) [9].

Previously, several studies suggested that probiotics modulate the cytokine profile in the cells through TLR stimulation (TLR-2 and TLR-4) [24, 25]. In this context, the studies on TLRs in immune recognition of immunomodulators have helped us to understand how probiotics function as an immunostimulator or immunosuppressor in immune cells. TLRs initiate and regulate the innate immune response through the NF- $\mathrm{kB}$ signalling pathway [26]. Thus, the TLRs are the key sensors of the probiotics for controlling many cellular activities in the innate immune response. One of our striking findings was that MHT-LM1004 remarkably activated macrophages by enhancing the TLR-2 expression levels, but not TLR-4, suggesting that the immunostimulating activity of MHTLM1004 was associated with the TLR-2 sensor. In accordance with our results, some reports demonstrated the role of TLR-2 in the sensing of Bifidobacteria and Lactobacilli [27-29].

Macrophage activation is an important strategy in the host defense response and immune enhancement. When macrophages are activated, a series of signalling molecules selectively turn on immunostimulatory genes including cytokines and iNOS through NF-KB [16]. Among these immune signalling pathways, the MAPK/NF- $\mathrm{KB}$ pathway is crucial for orchestrating early innate immune responses, which are essential for the host defense against a wide range of pathogens $[16,30]$. Consistently, our data indicated that upon treatment with MHT-LM1004, MAPK (ERK) phosphorylation was enhanced and NF- $\mathrm{KB}$ was translocated from the cytoplasm to the nucleus. These results revealed that MAPK/NF-кB signalling is involved in MHT-LM1004induced cytokine release and iNOS expression in RAW 264.7 macrophages.

Taken together, our study demonstrated that micronized and heat-treated L. plantarum LM1004 (MHT-LM1004) enhances immune responses both in vitro and in vivo through the activation of macrophages to secrete proinflammatory cytokines such as TNF- $\alpha$ and IL-6, induce iNOS expression, and up-regulate splenocyte proliferation. Notably, our study revealed that the TLR-2/MAPKs (ERK)/NF-KB pathway is a newly discovered signalling cascade related to probiotics, which functions in early innate immune responses. Therefore, we herein propose that the micronized and heat-treated L. plantarum LM1004 (MHT-LM1004) could function as an effective immunostimulator in early innate immune responses.

\section{Acknowledgments}

This work was supported by the Ministry of Trade, Industry, and Energy (MOTIE), Korea, under the "Regional Specialized Industry Development Program (Project No. R0005616)" supervised by the Korea Institute for Advancement of Technology (KIAT).

\section{Conflict of Interest}

The authors have no financial conflicts of interest to declare.

\section{References}

1. Hwang JH, Lim SB. 2017. Immunostimulatory activity of Opuntia ficus-indica var. Saboten cladodes fermented by Lactobacillus plantarum and Bacillus subtilis in RAW 264.7 macrophages. J. Med. Food 20: 131-139.

2. Adams CA. 2010. The probiotic paradox: live and dead cells are biological response modifiers. Nutr. Res. Rev. 23: 37-46.

3. Aoki-Yoshida A, Saito S, Tsuruta T, Ohsumi A, Tsunoda H, Sonoyama K. 2017. Exosomes isolated from sera of mice fed Lactobacillus strains affect inflammatory cytokine production in macrophages in vitro. Biochem. Biophys. Res. Commun. 489: 248-254.

4. Chuang L, Wu KG, Pai C, Hsieh PS, Tsai JJ, Yen JH, et al. 2007. Heat-killed cells of lactobacilli skew the immune response toward $\mathrm{T}$ helper 1 polarization in mouse splenocytes and dendritic cell-treated T cells. J. Agr. Food Chem. 55: 11080-11086.

5. He F, Morita H, Kubota A, Ouwehand AC, Hosoda M, Hiramatsu M, et al. 2005. Effect of orally administered nonviable Lactobacillus cells on murine humoral immune responses. Microbiol. Immunol. 49: 995-999.

6. Ou CC, Lin SL, Tsai JJ, Lin MY. 2011. Heat-killed lactic acid bacteria enhance immunomodulatory potential by skewing the immune response toward Th1 polarization. J. Food Sci. 76: M260-267. 
7. de Almada CN, Almada CN, Martinez RCR, Sant'Ana AS. 2016. Paraprobiotics: Evidences on their ability to modify biological responses, inactivation methods and perspectives on their application in foods. Trends Food Sci. Technol. 58: 96-114.

8. Sasaki E, Suzuki S, Fukui Y, Yajima N. 2015. Cell-bound exopolysaccharides of Lactobacillus brevis KB290 enhance cytotoxic activity of mouse splenocytes. J. Appl. Microbiol. 118: 506-514.

9. Lee HA, Kim H, Lee KW, Park KY. 2015. Dead nano-sized Lactobacillus plantarum inhibits azoxymethane/dextran sulfate sodium-induced colon cancer in Balb/c mice. J. Med. Food. 18: 1400-1405.

10. T. Kan, M. Ohwaki, Lactobacillus having ability to induce IL12 production, and method for culturing same, US Patents (2013)

11. Tabata Y, Inoue Y, Ikada Y. 1996. Size effect on systemic and mucosal immune responses induced by oral administration of biodegradable microspheres. Vaccine 14: 1677-1685.

12. Lee HA, Kim H, Lee KW, Park KY. 2016. Dead Lactobacillus plantarum stimulates and skews immune responses toward T helper 1 and 17 polarizations in RAW 264.7 cells and mouse splenocytes. J. Microbiol. Biotechnol. 26: 469-476.

13. Jung I, Lovitt RW. 2010. A comparative study of the growth of lactic acid bacteria in a pilot scale membrane bioreactor. J. Chem. Technol. Biotechnol. 85: 1250-1259.

14. Jung IS, Oh MK, Cho YC, Kong IS. 2011. The viability to a wall shear stress and propagation of Bifidobacterium longum in the intensive membrane bioreactor. Appl. Microbiol. Biotechnol. 92: 939-949.

15. Mosmann T. 1983. Rapid colorimetric assay for cellular growth and survival: application to proliferation and cytotoxicity assays. J. Immunol. Methods 65: 55-63.

16. Lee J, Choi JW, Sohng JK, Pandey RP, Park YI. 2016. The immunostimulating activity of quercetin 3-O-xyloside in murine macrophages via activation of the ASK1/MAPK/ NF-kappaB signaling pathway. Int. Immunopharmacol. 31: 88-97.

17. Chien AC, Hill NS, Levin PA. 2012. Cell size control in bacteria. Curr. Biol. 22: R340-349.

18. Vassalli P. 1992. The pathophysiology of tumor necrosis factors. Annu. Rev. Immunol. 10: 411-452.
19. Bogdan C. 2001. Nitric oxide and the immune response. Nat. Immunol. 2: 907-916.

20. Hirschfeld M, Ma Y, Weis JH, Vogel SN, Weis JJ. 2000. Cutting edge: repurification of lipopolysaccharide eliminates signaling through both human and murine toll-like receptor 2. J. Immunol. 165: 618-622.

21. Takeuchi O, Akira S. 2001. Toll-like receptors; their physiological role and signal transduction system. Int. Immunopharmacol. 1: 625-635.

22. Xie QW, Kashiwabara Y, Nathan C. 1994. Role of transcription factor NF-kappa B/Rel in induction of nitric oxide synthase. J. Biol. Chem. 269: 4705-4708.

23. Gill HS, Rutherfurd KJ, Prasad J, Gopal PK. 2000. Enhancement of natural and acquired immunity by Lactobacillus rhamnosus (HN001), Lactobacillus acidophilus (HN017) and Bifidobacterium lactis (HN019). Br. J. Nutr. 83: 167-176.

24. Galdeano CM, de Moreno de LeBlanc A, Vinderola G, Bonet ME, Perdigon G. 2007. Proposed model: mechanisms of immunomodulation induced by probiotic bacteria. Clin. Vaccine Immunol. 14: 485-492.

25. Dogi CA, Galdeano CM, Perdigon G. 2008. Gut immune stimulation by non pathogenic Gram(+) and Gram(-) bacteria. Comparison with a probiotic strain. Cytokine 41: 223-231.

26. Iwasaki A, Medzhitov R. 2004. Toll-like receptor control of the adaptive immune responses. Nat. Immunol. 5: 987-995.

27. van Bergenhenegouwen J, Kraneveld AD, Rutten L, Kettelarij N, Garssen J, Vos AP. 2014. Extracellular vesicles modulate host-microbe responses by altering TLR2 activity and phagocytosis. PLoS One 9: e89121.

28. Zeuthen LH, Fink LN, Frokiaer H. 2008. Toll-like receptor 2 and nucleotide-binding oligomerization domain-2 play divergent roles in the recognition of gut-derived lactobacilli and bifidobacteria in dendritic cells. Immunology 124: 489-502.

29. Plantinga TS, van Maren WW, van Bergenhenegouwen J, Hameetman M, Nierkens S, Jacobs C, et al. 2011. Differential Toll-like receptor recognition and induction of cytokine profile by Bifidobacterium breve and Lactobacillus strains of probiotics. Clin. Vaccine Immunol. 18: 621-628.

30. Senthil Kumar KJ, Wang SY. 2009. Lucidone inhibits iNOS and COX-2 expression in LPS-induced RAW 264.7 murine macrophage cells via NF-kappaB and MAPKs signaling pathways. Planta Med. 75: 494-500. 